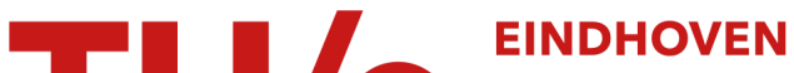 UNIVERSITY OF TECHNOLOGY
}

\section{Cryptanalysis of reduced versions of the HIGHT block cipher from CHES 2006}

\section{Citation for published version (APA):}

Lu, J. (2007). Cryptanalysis of reduced versions of the HIGHT block cipher from CHES 2006. In K. H. Nam, \& G. Rhee (Eds.), Proceedings of the 10th International Conference on Information Security and Cryptology (ICISC 2007) 29-30 November 2007, Seoul, Korea (pp. 11-26). (Lecture Notes in Computer Science; Vol. 4817). Springer. https://doi.org/10.1007/978-3-540-76788-6_2

DOI:

10.1007/978-3-540-76788-6_2

Document status and date:

Published: 01/01/2007

\section{Document Version:}

Publisher's PDF, also known as Version of Record (includes final page, issue and volume numbers)

\section{Please check the document version of this publication:}

- A submitted manuscript is the version of the article upon submission and before peer-review. There can be important differences between the submitted version and the official published version of record. People interested in the research are advised to contact the author for the final version of the publication, or visit the $\mathrm{DOI}$ to the publisher's website.

- The final author version and the galley proof are versions of the publication after peer review.

- The final published version features the final layout of the paper including the volume, issue and page numbers.

Link to publication

\section{General rights}

Copyright and moral rights for the publications made accessible in the public portal are retained by the authors and/or other copyright owners and it is a condition of accessing publications that users recognise and abide by the legal requirements associated with these rights.

- Users may download and print one copy of any publication from the public portal for the purpose of private study or research.

- You may not further distribute the material or use it for any profit-making activity or commercial gain

- You may freely distribute the URL identifying the publication in the public portal.

If the publication is distributed under the terms of Article $25 \mathrm{fa}$ of the Dutch Copyright Act, indicated by the "Taverne" license above, please follow below link for the End User Agreement:

www.tue.nl/taverne

Take down policy

If you believe that this document breaches copyright please contact us at:

openaccess@tue.nl

providing details and we will investigate your claim. 


\title{
Cryptanalysis of Reduced Versions of the HIGHT Block Cipher from CHES 2006*
}

\author{
Jiqiang Lu \\ Information Security Group, Royal Holloway, University of London \\ Egham, Surrey TW20 0EX, UK \\ lvjiqiang@hotmail.com
}

\begin{abstract}
HIGHT is a 32-round block cipher with a 64 -bit block size and a 128-bit user key, which was proposed at CHES '06 for low-resource applications like RFID. In this paper, we present an impossible differential attack on 25-round HIGHT, a related-key rectangle attack on 26round HIGHT, and finally a related-key impossible differential attack on 28-round HIGHT. Our result suggests that the safety margin of HIGHT decreases from the originally expected thirteen rounds to about four rounds now.
\end{abstract}

Keywords: Block cipher, HIGHT, Impossible differential cryptanalysis, Rectangle attack, Related-key attack.

\section{Introduction}

Recently, cryptography for embedded and ubiquitous computing systems receives an extensive research attention. At CHES '06, Hong et al. 9] presented a 32-round block cipher with a 64-bit block size and a 128-bit user key, known as HIGHT. Due to the simple byte-oriented operations involved, HIGHT is especially efficient in hardware implementations, much faster than those 78 of AES [19, and it is most suitable for various real-life resource-constrained application environments, such as RFID (Radio Frequency Identification) systems. The HIGHT proposers also analysed its security against various existing cryptanalytic attacks; they described a differential attack [6], a linear attack [18] and a boomerang attack 20] on 13-round HIGHT, a truncated differential attack [14] and a saturation attack [17] on 16-round HIGHT, an impossible differential attack 215] on 18-round HIGHT, and finally a related-key [112] boomerang attack [5] on 19-round HIGHT.

In this paper, we further analyse the security of HIGHT. We exploit 16-round impossible differentials such that we can devise an impossible differential attack on 25-round HIGHT; we also exploit 18-round related-key rectangle distinguishers with probability $2^{-92.4}$, which can enable us to mount a related-key rectangle

\footnotetext{
* This work as well as the author was supported by a British Chevening / Royal Holloway Scholarship and the European Commission under contract IST-2002-507932 (ECRYPT).
} 
attack on 26-round HIGHT. Finally, we exploit 19-round related-key impossible differentials that can be used to mount a related-key impossible differential attack on 28-round HIGHT.

The rest of this paper is organised as follows. In the next section, we briefly describe some notation and the HIGHT block cipher. In Sections 3 and 4, we present our cryptanalytic results. Section [5]concludes this paper.

\section{Preliminaries}

\subsection{Notation}

We will use the following notation throughout this paper.

$-\oplus$ : bitwise logical exclusive OR (XOR)

- $\boxplus$ : addition modulo $2^{8}$

- $i$ : left rotation by $i$ bits

$-e_{j}$ : a byte with zeros in all positions but bit $j,(0 \leq j \leq 7)$

$-e_{i_{1}, \cdots, i_{j}}: e_{i_{1}} \oplus \cdots \oplus e_{i_{j}},\left(0 \leq i_{1}, \cdots, i_{j} \leq 7\right)$

$-e_{j, \sim}$ : a byte that has zeros in bits 0 to $j-1$, a one in bit $j$ and indeterminate values in bits $(j+1)$ to 7

$-e_{\bar{j}, \sim}$ : a byte that has zeros in bits 0 to $j$ and indeterminate values in bits $(j+1)$ to 7

- ? : an arbitrary byte, where two bytes represented by the ? symbol may be different

The notion of difference used throughout this paper is with respect to the $\oplus$ operation. It is assumed that in a byte the rightmost bit is the least significant bit and referred as the 0 -th bit, and the leftmost bit is the most significant bit and referred as the 7 -th bit.

\subsection{The HIGHT Block Cipher}

HIGHT [9] takes as an input a 64-bit plaintext $P$, represented as eight bytes $\left(P_{7}, \cdots, P_{1}, P_{0}\right)$, and it has a total of 32 rounds. Let $\left(X_{i-1,7}, X_{i-1,6}, X_{i-1,5}, X_{i-1,4}\right.$, $\left.X_{i-1,3}, X_{i-1,2}, X_{i-1,1}, X_{i-1,0}\right)$ denote the eight-byte input to Round $i$, and $\left(X_{i, 7}\right.$, $\left.X_{i, 6}, X_{i, 5}, X_{i, 4}, X_{i, 3}, X_{i, 2}, X_{i, 1}, X_{i, 0}\right)$ denote the eight-byte output of Round $i$, $(1 \leq i \leq 32)$. The encryption procedure can be described as follows.

1. Perform the Initial Transformation: the eight-byte output $\left(X_{0,7}, X_{0,6}, X_{0,5}\right.$, $\left.X_{0,4}, X_{0,3}, X_{0,2}, X_{0,1}, X_{0,0}\right)=\left(P_{7}, P_{6} \oplus \mathrm{WK}_{3}, P_{5}, P_{4} \boxplus \mathrm{WK}_{2}, P_{3}, P_{2} \oplus \mathrm{WK}_{1}, P_{1}\right.$, $\left.P_{0} \boxplus \mathrm{WK}_{0}\right)$.

2. For $i=1$ to 32 :

$X_{i, 0}=X_{i-1,7} \oplus\left(F_{0}\left(X_{i-1,6}\right) \boxplus \mathrm{SK}_{4 i-1}\right)$,

$X_{i, 1}=X_{i-1,0}$,

$X_{i, 2}=X_{i-1,1} \boxplus\left(F_{1}\left(X_{i-1,0}\right) \oplus \mathrm{SK}_{4 i-2}\right)$,

$X_{i, 3}=X_{i-1,2}$,

$X_{i, 4}=X_{i-1,3} \oplus\left(F_{0}\left(X_{i-1,2}\right) \boxplus \mathrm{SK}_{4 i-3}\right)$, 


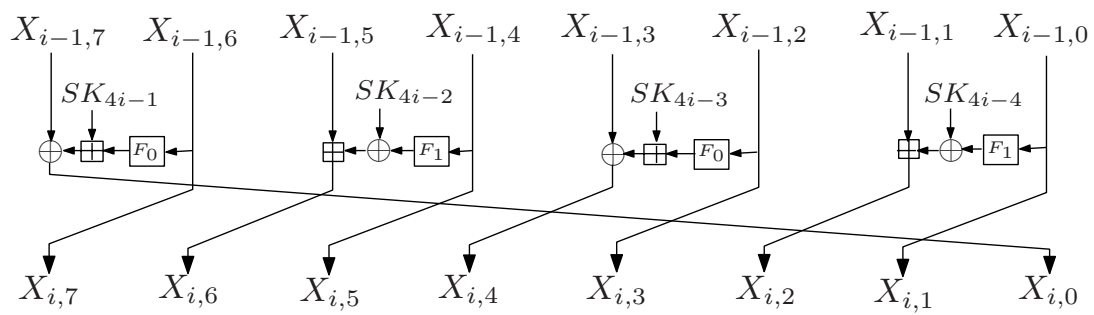

Fig. 1. The $i$-th encryption round of HIGHT

$$
\begin{aligned}
& X_{i, 5}=X_{i-1,4}, \\
& X_{i, 6}=X_{i-1,5} \boxplus\left(F_{1}\left(X_{i-1,4}\right) \oplus \mathrm{SK}_{4 i-4}\right), \\
& X_{i, 7}=X_{i-1,6} .
\end{aligned}
$$

3. Perform the Final Transformation: the ciphertext $C=\left(C_{7}, C_{6}, C_{5}, C_{4}, C_{3}, C_{2}\right.$, $\left.C_{1}, C_{0}\right)=\left(X_{32,0}, X_{32,7} \oplus \mathrm{WK}_{7}, X_{32,6}, X_{32,5} \boxplus \mathrm{WK}_{6}, X_{32,4}, X_{32,3} \oplus \mathrm{WK}_{5}, X_{32,2}\right.$, $\left.X_{32,1} \boxplus \mathrm{WK}_{4}\right)$.

In the above description, $\mathrm{SK}_{i}(0 \leq i \leq 127)$ are the round subkeys, $\mathrm{WK}_{j}(0 \leq$ $j \leq 7)$ are the whitening subkeys used in the initial and final transformation, the functions $F_{0}(\cdot)$ and $F_{1}(\cdot)$ are defined as $F_{0}(x)=(x \lll 1) \oplus(x \lll 2) \oplus(x \lll 7)$, and $F_{1}(x)=(x \lll 3) \oplus(x \lll 4) \oplus(x \lll 6)$. Note that the first round is referred as Round 1. Fig. 1 depicts one encryption round of HIGHT.

The key schedule of HIGHT only accepts a 128-bit user key MK, represented as sixteen bytes $\left(\mathrm{MK}_{15}, \cdots, \mathrm{MK}_{1}, \mathrm{MK}_{0}\right)$. The whitening subkeys $\mathrm{WK}_{j}$ are generated as follows: $\mathrm{WK}_{j}=\mathrm{MK}_{j+12}$ for $j=0,1,2,3$, and $\mathrm{WK}_{j}=\mathrm{MK}_{j-4}$ for $j=$ $4,5,6,7$. The round subkeys are generated as follows: $\mathrm{SK}_{16 \cdot i+j}=\mathrm{MK}_{j-i \bmod 8} \boxplus$ $\delta_{16 \cdot i+j}$, or $\mathrm{SK}_{16 \cdot i+j+8}=\mathrm{MK}_{(j-i \bmod 8)+8} \boxplus \delta_{16 \cdot i+j+8}(0 \leq i, j \leq 7)$, where $\delta_{16 \cdot i+j}$ and $\delta_{16 \cdot i+j+8}$ are public constants.

\section{Impossible Differential Attack on 25-Round HIGHT}

\subsection{6-Round Impossible Differentials}

We exploit certain 16-round impossible differentials: $\left(e_{i, \sim}, 0,0,0,0,0,0,0\right) \nrightarrow$ $\left(e_{0,3,5,6,7}, 0,0,0,0,0,0, e_{7}\right)$, where $1 \leq i \leq 7$. Note that the 16 -round differentials $\left(e_{7}, e_{0,3,5,6,7}, 0,0,0,0,0,0\right) \rightarrow\left(0, e_{i, \sim}, 0,0,0,0,0,0\right)$ are also impossible. These 16 round impossible differentials are mainly because the following general property.

Property 1. The $\boxplus$ operation definitely preserves the least significant differences in the original positions, and may preserve the other differences in the original positions or propagate them to the more significant positions, but never to the less significant positions, while the $\oplus$ operation always preserves all the differences in their original positions.

The 16-round impossible differentials are built in a miss-in-the-middle manner [3]: a 8-round differential $\left(e_{i, \sim}, 0,0,0,0,0,0,0\right) \rightarrow\left(e_{i, \sim}, ?, ?, ?, ?, ?, ?, ?\right)$ with 
probability 1 is concatenated with another 8-round differential $\left(e_{0, \sim}, 0, ?, ?, ?, ?, ?\right.$, $?) \leftarrow\left(e_{0,3,5,6,7}, 0,0,0,0,0,0, e_{7}\right)$ with probability 1 , but the leftmost bytes of the intermediate differences of these two differentials contradict one another. The input difference $\left(e_{i, \sim}, 0,0,0,0,0,0,0\right)$ of the first 8-round differential propagates to a difference $\left(0,0,0,0,0,0,0, e_{i, \sim}\right)$ after one round of HIGHT, which then propagates to a difference $\left(0,0,0,0,0, ?, e_{i, \sim}, 0\right)$ after another round. As a result, the difference $\left(0,0,0,0,0, ?, e_{i, \sim}, 0\right)$ finally propagates to a difference $\left(e_{i, \sim}, ?, ?, ?, ?, ?, ?, ?\right)$ after the following six rounds. On the other hand, when we roll back the difference $\left(e_{0,3,5,6,7}, 0,0,0,0,0,0, e_{7}\right)$ through one round of HIGHT in the reverse direction, then we will definitely get the difference $\left(0, e_{0,3,5,6,7}, 0,0\right.$, $0,0,0,0)$, as the difference $e_{0,3,5,6,7}$ becomes $\left(e_{0,3,5,6,7} \lll 1\right) \oplus\left(e_{0,3,5,6,7} \lll 2\right) \oplus$ $\left(e_{0,3,5,6,7} \lll 7\right)=e_{0,1,4,6,7} \oplus e_{0,1,2,5,7} \oplus e_{2,4,5,6,7}=e_{7}$ after the $F_{0}$ function. The difference $\left(0, e_{0,3,5,6,7}, 0,0,0,0,0,0\right)$ propagates to a difference $\left(e_{0, \sim}, 0, ?, ?, ?, ?, ?\right.$, ?) when we roll it back through seven more rounds. Now a contradiction occurs if $i \neq 0$, as the leftmost byte difference of one of the two intermediate differences is $e_{i, \sim}$ while the leftmost byte difference of the other is $e_{0, \sim}$.

\subsection{Attacking Rounds 6-30}

HIGHT has a Feistel structure with four branches, which can be efficiently implemented. However, we observe this round structure is much weaker than a regular Feistel structure, in terms of security.

Property 2. A byte value (or difference) of the input to Round $i$ will affect at most two bytes of the output of Round $i$, at most four bytes of the output of Round $(i+1)$, and at most six bytes of the output of Round $(i+2),(1 \leq i \leq 29)$.

Property 2 suggests that to get a byte value (or difference) of the input to a round we need not guess all the twelve 8-bit subkeys in its following three rounds; and we can determine whether a candidate pair is a right pair byte by byte, and even bit by bit, due to the round struture and the operations involved. This plays an important role in our attacks.

We can use the 16-round impossible differentials to break 25-round HIGHT. Here, we attack Rounds 6 to 30 of HIGHT with the final transformation only. The attack procedure is as follows.

1. Choose $2^{13}$ structures of $2^{47}$ plaintexts, where the two bytes $(0,1)$ and the least significant bits of the third bytes of the $2^{47}$ plaintexts in a structure are fixed to certain values, and the other 47 bit positions take all the possible values. Obviously, a structure proposes $2^{47 \times 2} / 2=2^{93}$ plaintext pairs $\left(P^{i}, P^{j}\right)$ with difference $\left(?, ?, ?, ?, ?, e_{\overline{0}, \sim}, 0,0\right),\left(i, j=1,2, \cdots, 2^{47}\right)$, thus the $2^{13}$ structures propose a total of $2^{106}$ plaintext pairs.

2. In a chosen-plaintext attack scenario, obtain all the ciphertexts $C^{i}$ of the plaintexts $P^{i}$. Choose only the ciphertext pairs $\left(C^{i}, C^{j}\right)$ with difference $\left(?, ?, ?, ?, ?, e_{0, \sim}, 0,0\right)$.

3. Guess the two key bytes $\left(\mathrm{MK}_{0}, \mathrm{MK}_{3}\right)$, compute the subkeys $\left(\mathrm{WK}_{7}, \mathrm{SK}_{119}\right)$ in the final transformation and Round 30, and do the following. 
(a) Partially decrypt every remaining ciphertext pair $\left(C^{i}, C^{j}\right)$ with $\left(\mathrm{WK}_{7}\right.$, $\left.\mathrm{SK}_{119}\right)$ to get the two bytes $(7,6)$ of their intermediate values just before Round 30, and check if they have a difference $(0, ?)$. Keep only the qualified pairs.

(b) Guess the two key bytes $\left(\mathrm{MK}_{2}, \mathrm{MK}_{7}\right)$, compute the subkeys $\left(\mathrm{WK}_{6}, \mathrm{SK}_{118}\right)$ in the final transformation and Round 30, and compute the subkey $\mathrm{SK}_{114}$ in Round 29 with $\mathrm{MK}_{3}$ guessed above. Partially decrypt every remaining $\left(C^{i}, C^{j}\right)$ with $\left(\mathrm{WK}_{6}, \mathrm{SK}_{114}, \mathrm{SK}_{118}\right)$ to get the two bytes $(5,4)$ of their intermediate values just before Round 291 Check if they have a difference $(0, ?)$. Keep only the qualified pairs.

(c) Guess the 8 key bits $\mathrm{MK}_{1}$, compute the subkey $\mathrm{WK}_{5}$ in the final transformation, and do as follows.

i. Guess the least significant bit $\mathrm{MK}_{6,0}$ of the key byte $\mathrm{MK}_{6}$, and compute the least significant bit $\mathrm{SK}_{117,0}$ of the subkey $\mathrm{SK}_{117}$ in Round 30. Partially decrypt every remaining $\left(C^{i}, C^{j}\right)$ with $\left(\mathrm{WK}_{5}, \mathrm{SK}_{117,0}\right)$ to get their intermediate values $\left(X_{29,3,0}^{i}, X_{29,2}^{i}\right)$ and $\left(X_{29,3,0}^{j}, X_{29,2}^{j}\right)$ just before Round 30. Keep the pairs such that $X_{29,3,0}^{i} \oplus X_{29,3,0}^{j}=1$.

ii. Guess the other seven bits $\mathrm{MK}_{6,1-7}$ of $\mathrm{MK}_{6}$, and compute the subkey $\mathrm{SK}_{117}$ (together with $\mathrm{MK}_{6,0}$ guessed above). Partially decrypt every remaining $\left(C^{i}, C^{j}\right)$ with $\left(\mathrm{WK}_{5}, \mathrm{SK}_{117}\right)$ to get the two bytes $(2,3)$ of their intermediate values just before Round 30 .

(d) Compute the subkey $\mathrm{SK}_{113}$ in Round 29 with $\mathrm{MK}_{2}$ guessed above. For every remaining $\left(C^{i}, C^{j}\right)$, partially decrypt the two bytes $(4,3)$ of their intermediate values just before Round 30 with $\mathrm{SK}_{113}$ to get the two bytes $(3,2)$ of their intermediate values just before Round 29 . Check if they have a difference $\left(e_{2, \sim}, e_{0, \sim}\right)$. Keep only the qualified pairs.

(e) For $l=0$ to 7 , do as follows.

- Guess the $l$-th bit $\mathrm{MK}_{15, l}$ of the key byte $\mathrm{MK}_{15}$, and compute the $(l+1)$ bits $\mathrm{SK}_{109,0-l}$ of the subkey $\mathrm{SK}_{109}$ in Round 28.

- For every remaining $\left(C^{i}, C^{j}\right)$, partially decrypt the two bytes $(4,3)$ of their intermediate values just before Round 29 with $\mathrm{SK}_{109,0-l}$ to get their intermediate values $\left(X_{27,3,0-l}^{i}, X_{27,2}^{i}\right)$ and $\left(X_{27,3,0-l}^{j}, X_{27,2}^{j}\right)$ just before Round 28. Keep the pairs such that $X_{27,3,0-l}^{i}=X_{27,3,0-l}^{j}$.

(f) Guess the 8 key bits $\mathrm{MK}_{5}$, compute the subkey $\mathrm{SK}_{116}$ in Round 30, and compute the subkeys $\left(\mathrm{WK}_{4}, \mathrm{SK}_{112}\right)$ in the final transformation and Round 29 with $\left(\mathrm{MK}_{0}, \mathrm{MK}_{1}\right)$ guessed above. Partially every remaining $\left(C^{i}, C^{j}\right)$ with $\left(\mathrm{WK}_{4}, \mathrm{SK}_{112}, \mathrm{SK}_{116}\right)$ to get the two bytes $(1,0)$ of their intermediate values just before Round 29. Check if they have the difference $\left(e_{0,3,5,6,7}, 0\right)$. Keep only the qualified pairs.

(g) Guess the least significant bit $\mathrm{MK}_{14,0}$ of the key byte $\mathrm{MK}_{14}$; for $l=1$ to 7 , do as follows.

${ }^{1}$ The other required intermediate values have been obtained in the previous steps. Same for some following steps as well as the attacks in the next section, without explicit statement. 
- Guess the $l$-th bit $\mathrm{MK}_{14, l}$ of the key byte $\mathrm{MK}_{14}$, and compute the $(l+1)$ bits $\mathrm{SK}_{108,0-l}$ of the subkey $\mathrm{SK}_{108}$ in Round 28.

- For every remaining $\left(C^{i}, C^{j}\right)$, partially decrypt the two bytes $(1,2)$ of their intermediate values just before Round 29 with $\mathrm{SK}_{108,0-l}$ to get their intermediate values $\left(X_{27,1,0-l}^{i}, X_{27,1,0-l}^{j}\right)$ just before Round 28. If $l \neq 7$, keep the pairs such that $X_{27,1,0-l}^{i}=X_{27,1,0-l}^{j}$; if $l=7$, keep the pairs $X_{27,1,0-l}^{i} \oplus X_{27,1,0-l}^{j}=e_{7}$.

(h) Guess the least significant 3 bits $\mathrm{MK}_{10,0-2}$ of the key byte $\mathrm{MK}_{10}$; for $l=3$ to 7 , do as follows.

- Guess the $l$-th bit $\mathrm{MK}_{10, l}$ of the key byte $\mathrm{MK}_{10}$, and compute the $(l+1)$ bits $\mathrm{SK}_{104,0-l}$ of the subkey $\mathrm{SK}_{104}$ in Round 27.

- For every remaining $\left(C^{i}, C^{j}\right)$, partially decrypt the two bytes $(1,2)$ of their intermediate values just before Round 28 with $\mathrm{SK}_{104,0-l}$ to get their intermediate values $\left(X_{26,1,0-l}^{i}, X_{26,1,0-l}^{j}\right)$ just before Round 27. Keep the pairs such that $X_{26,1,0-l}^{i}=X_{26,1,0-l}^{j}$.

4. Compute the subkey $\mathrm{SK}_{23}$ with $\mathrm{MK}_{6}$ guessed in Step 3, and do the following.

(a) Partially encrypt every plaintext pair $\left(P^{i}, P^{j}\right)$ corresponding to a remaining ciphertext pair $\left(C^{i}, C^{j}\right)$, with $\mathrm{SK}_{23}$ to get the two bytes $(7,0)$ of their intermediate values just after Round 6 . Check if they have a difference $(?, 0)$. Keep only the qualified pairs.

(b) Compute the subkeys $\left(\mathrm{SK}_{22}, \mathrm{SK}_{27}\right)$ with $\left(\mathrm{MK}_{5}, \mathrm{MK}_{10}\right)$ guessed in Step 3. Partially encrypt every remaining $\left(P^{i}, P^{j}\right)$ with $\left(\mathrm{SK}_{22}, \mathrm{SK}_{27}\right)$ to get the two bytes $(7,0)$ of their intermediate values just after Round 7 . Check if they have a difference $(?, 0)$. Keep only the qualified pairs.

(c) Guess the two key bytes $\left(\mathrm{MK}_{4}, \mathrm{MK}_{9}\right)$, compute the subkeys $\left(\mathrm{SK}_{21}, \mathrm{SK}_{26}\right)$ in Rounds 6 and 7, and compute the subkey $\mathrm{SK}_{31}$ with $\mathrm{MK}_{14}$ guessed in Step 3. Partially encrypt every remaining $\left(P^{i}, P^{j}\right)$ with $\left(\mathrm{SK}_{21}, \mathrm{SK}_{26}, \mathrm{SK}_{31}\right)$ to get the two bytes $(7,0)$ of their intermediate values just after Round 8 . Check if they have a difference $(?, 0)$. Keep only the qualified pairs.

(d) Guess the two key bytes $\left(\mathrm{MK}_{8}, \mathrm{MK}_{13}\right)$, compute the subkeys $\left(\mathrm{SK}_{25}, \mathrm{SK}_{30}\right)$ in Rounds 7 and 8 , and compute the subkeys $\left(\mathrm{SK}_{20}, \mathrm{SK}_{35}\right)$ with $\left(\mathrm{MK}_{1}\right.$, $\left.\mathrm{MK}_{3}\right)$ guessed in Step 3. Partially encrypt every remaining $\left(P^{i}, P^{j}\right)$ with $\left(\mathrm{SK}_{20}, \mathrm{SK}_{25}, \mathrm{SK}_{30}, \mathrm{SK}_{35}\right)$ to get the two bytes $(7,0)$ of their intermediate values just after Round 9. Check if they have a difference (?,0). Keep only the qualified pairs.

(e) Guess the key byte $\mathrm{MK}_{12}$, compute the subkey $\mathrm{SK}_{29}$, and compute the subkeys $\left(\mathrm{SK}_{24}, \mathrm{SK}_{34}, \mathrm{SK}_{39}\right)$ with $\left(\mathrm{MK}_{0}, \mathrm{MK}_{5}, \mathrm{MK}_{15}\right)$ guessed in Step 3. Partially encrypt every remaining $\left(P^{i}, P^{j}\right)$ with $\left(\mathrm{SK}_{24}, \mathrm{SK}_{29}, \mathrm{SK}_{34}, \mathrm{SK}_{39}\right)$ to get the two bytes $(7,0)$ of their intermediate values just after Round 10 . Check if they have a difference $\left(e_{\overline{0}, \sim}, 0\right)$. If none of the plaintext pairs satisfies this test, record the guessed 120 key bits $\left(\mathrm{MK}_{0}, \cdots, \mathrm{MK}_{10}, \mathrm{MK}_{12}\right.$, $\left.\cdots, \mathrm{MK}_{15}\right)$, and execute Step 5; otherwise, discard this guess and try another.

5. For a recorded $\left(\mathrm{MK}_{0}, \cdots, \mathrm{MK}_{10}, \mathrm{MK}_{12}, \cdots, \mathrm{MK}_{15}\right)$, exhaustively search for the remaining 8 key bits with three known pairs of plaintexts and ciphertexts. 
If a 128-bit key is suggested, output it as the user key of the 25-round HIGHT; otherwise, go to Step 3.

There are 17-bit, 8-bit, 8-bit, 1-bit, 3-bit and 7-bit filtering conditions over the ciphertext pairs in Steps 2 and $3-(\mathrm{a}) \sim(\mathrm{d})$ and $(\mathrm{f})$, respectively, and a 1bit filtering condition in every iteration of Steps 3-(e), (g) and (h). Thus, it is expected about $2^{106} \cdot 2^{-64}=2^{42}$ ciphertext pairs remain after Step 3 . There is a 8-bit filtering condition in each of Steps 4 -(a) (e), so it follows that about $2^{120}$. $\left(1-2^{-8}\right)^{2^{10}} \approx 2^{120} \cdot e^{-2^{2}} \approx 2^{114.24}$ guesses of the 120 key bits are recorded in Step 4-(e). The probability that a wrong key is suggested in Step 5 is approximately $2^{-64 \times 3}=2^{-192}$, thus, the expected number of wrong keys in Step 5 is about $2^{-192} \cdot 2^{114.24+8}=2^{-73.76}$. It is very likely that we can find the correct key.

The attack requires $2^{60}$ chosen plaintexts. Step 3 has about $2 \cdot 2^{89} \cdot 2^{16} \cdot \frac{1}{4}$. $\frac{1}{25}+2 \cdot 2^{81} \cdot 2^{32} \cdot \frac{1}{4} \cdot \frac{2}{25}+2 \cdot 2^{73} \cdot 2^{41} \cdot \frac{1}{4} \cdot \frac{1}{25}+2 \cdot 2^{72} \cdot 2^{48} \cdot \frac{1}{4} \cdot \frac{1}{25}+2 \cdot 2^{72} \cdot 2^{48}$. $\frac{1}{4} \cdot \frac{1}{25}+\sum_{l=0}^{7}\left(2 \cdot 2^{69-l} \cdot 2^{48+l+1} \cdot \frac{1}{4} \cdot \frac{1}{25}\right)+2 \cdot 2^{61} \cdot 2^{64} \cdot \frac{1}{4} \cdot \frac{1}{25}+\sum_{l=0}^{6}\left(2 \cdot 2^{54-l}\right.$. $\left.2^{64+2+l} \cdot \frac{1}{4} \cdot \frac{1}{25}\right)+\sum_{l=0}^{4}\left(2 \cdot 2^{47-l} \cdot 2^{72+4+l} \cdot \frac{1}{4} \cdot \frac{1}{25}\right) \approx 2^{120.73}$ computations. Step 4 has about $2 \cdot 2^{42} \cdot 2^{80} \cdot \frac{1}{4} \cdot \frac{1}{25}+2 \cdot 2^{34} \cdot 2^{80} \cdot \frac{1}{4} \cdot \frac{2}{25}+2 \cdot 2^{26} \cdot 2^{96} \cdot \frac{1}{4} \cdot \frac{3}{25}+2 \cdot$ $2^{18} \cdot 2^{112} \cdot \frac{1}{4} \cdot \frac{4}{25}+2 \cdot 2^{120}\left[1+\left(1-2^{-8}\right)+\cdots+\left(1-2^{-8}\right)^{2^{10}}\right] \cdot \frac{1}{4} \cdot \frac{4}{25} \approx 2^{126.68}$ computations. Step 5 has about $2^{122.24}$ computations. Therefore, the attack has a total time complexity of about $2^{126.75} 25$-round HIGHT computations.

\section{Related-Key Cryptanalysis of Reduced HIGHT}

A related-key attack 112 assumes that the attacker knows the differences between one or more pairs of unknown keys. In this section, we present a related-key rectangle attack on 26-round HIGHT, and a related-key impossible differential attack on 28-round HIGHT.

\subsection{Related-Key Rectangle Attack on 26-Round HIGHT}

A related-key rectangle attack [51013] is a combination of a related-key attack and a rectangle attack [4; it is based on a related-key rectangle distinguisher, which treats a block cipher $E:\{0,1\}^{n} \times\{0,1\}^{k} \rightarrow\{0,1\}^{n}$ as a cascade of two sub-ciphers $E=E^{1} \circ E^{0}$.

18-Round Related-Key Rectangle Distinguishers of HIGHT. Let $E^{0}$ denote Rounds 3 to 12 , and $E^{1}$ denote Rounds 13 to 20. The first related-key differential for this 18-round distinguisher is the following 10-round related-key differential $\alpha \rightarrow \beta$ with probability $2^{-12}$ for $E^{0}:\left(e_{1,3,5}, e_{0,1,6}, e_{7}, 0,0,0,0,0\right) \rightarrow$ $\left(0, e_{1,5,6}, e_{0,6,7}, e_{7}, 0,0,0,0\right) 2$, where the user key difference $K_{A} \oplus K_{B}=K_{C} \oplus$ $K_{D}=\left(\Delta \mathrm{MK}_{15}, \cdots, \Delta \mathrm{MK}_{1}, \Delta \mathrm{MK}_{0}\right)$ is $\left(0, \cdots, 0, e_{7}, 0,0\right)$. The second related-key

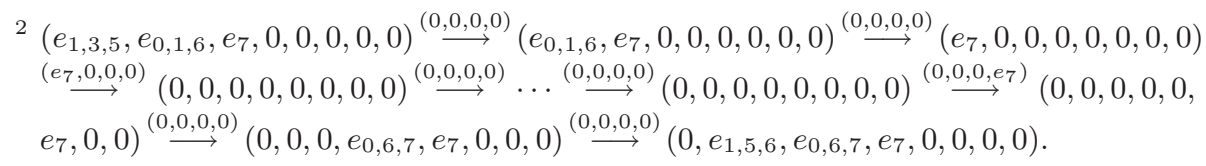


differential is the following 8-round related-key differential $\gamma \rightarrow \delta$ with probability $2^{-9}$ for $E^{1}:\left(0,0,0,0, e_{2,5,6}, e_{0,6,7}, e_{7}, 0\right) \rightarrow\left(e_{7}, 0,0,0,0,0,0, e_{0,1,6}\right)$, where the user key difference $K_{A} \oplus K_{C}=K_{B} \oplus K_{D}=\left(0, e_{7}, 0, \cdots, 0\right)$.

We can compute a square sum of at least $6 \cdot\left(2^{-12}\right)^{2}+20 \cdot\left(2^{-13}\right)^{2}+20 \cdot\left(2^{-14}\right)^{2}+$ $72 \cdot\left(2^{-15}\right)^{2} \approx 2^{-19.98}$ for the probabilities of all the possible 10-round relatedkey differentials $\alpha \rightarrow \beta^{\prime}$ for $E^{0}$, as there are at least 6 (10-round related-key differential characteristics) with probability $2^{-12}$, at least 20 with probability $2^{-13}$, at least 20 with probability $2^{-14}$, and at least 72 with probability $2^{-15}$. We can also compute a square sum of at least $5 \cdot\left(2^{-9}\right)^{2}+18 \cdot\left(2^{-10}\right)^{2}+40$. $\left(2^{-11}\right)^{2} \approx 2^{-14.42}$ for the probabilities of all the possible 8-round related-key differentials $\gamma^{\prime} \rightarrow \delta$ for $E^{1}$, as there are at least 5 (8-round related-key differential characteristics) with probability $2^{-9}$, at least 18 with probability $2^{-10}$, and at least 40 with probability $2^{-11}$.

Therefore, we can learn that this 18-round related-key rectangle distinguisher has a probability of at least $2^{-19.98} \cdot 2^{-14.42} \cdot 2^{-64}=2^{-98.4}$ for the correct key, while it has a probability of $2^{-128}$ for a wrong key. We can further improve it by counting many possible 8-round related-key differentials $\gamma^{\prime} \rightarrow \delta^{\prime}$ for every $\gamma^{\prime} \rightarrow \delta$ for $E^{1}$. We count those that only have the output difference $\left(e_{7}, 0,0,0,0,0,0, \Delta X_{21,0}\right)$ different from the 8-round differential $\gamma^{\prime} \rightarrow \delta$; an analysis of this one-round differentials reveals that there are 4 possible $\Delta X_{21,0}$ (i.e., $\left.e_{0,1,6}, e_{0,6}, e_{0,6,7}, e_{0,1,6,7}\right)$ with probability $2^{-3}, 4$ possible $\Delta X_{21,0}$ with probability $2^{-4}, 4$ possible $\Delta X_{21,0}$ with probability $2^{-5}, 4$ possible $\Delta X_{21,0}$ with probability $2^{-6}$, and 8 possible $\Delta X_{21,0}$ with probability $2^{-7}$. Actually, these are all the 24 possible output differences of the last one-round differentials; we denote them by the set $\mathcal{S}$. As a result, the distinguisher now has a probability of at least $2^{-19.98} \cdot\left(4 \cdot 2^{-7.21}+4 \cdot 2^{-8.21}+4 \cdot 2^{-9.21}+4 \cdot 2^{-10.21}+8 \cdot 2^{-11.21}\right)^{2} \cdot 2^{-64}=2^{-92.4}$ for the correct key, while it has a probability of $\left(24 \cdot 2^{-64}\right)^{2} \approx 2^{-118.83}$ for a wrong key. Similar related-key rectangle distinguishers exist for some other series of 18 rounds.

Attacking Rounds 1-26. To get the difference $\left(e_{1,3,5}, e_{0,1,6}, e_{7}, 0,0,0,0,0\right)$ just before Round 3, the input difference to Round 1 must have the form $\left(?, e_{0, \sim}, ?, e_{0, \sim}, e_{7}, 0,0,0\right)$, with 31 bits definitely being zero differences. On the other hand, the output difference $\left(e_{7}, 0,0,0,0,0,0, x\right)$ of this distinguisher will propagate to a difference $\left(0,0,0,0,0, ?, x, e_{7}\right)$ just after Round 21 , where $x \in \mathcal{S}$, which will then propagate to a difference $\left(0,0,0, ?, ?, e_{0, \sim}, e_{7}, 0\right)$ just after Round 22 , to a difference $\left(0, ?, ?, ?, e_{0, \sim}, e_{7}, 0, e_{7}\right)$ just after Round 23 (due to the sub-

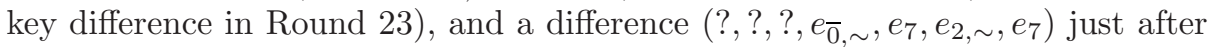
Round 24. This property allows us to use the early abort technique [16] to break Rounds 21 and 24; the main idea of the early abort technique is to partially determine whether or not a candidate quartet in a rectangle attack is valid earlier than usual; if not, we can discard it immediately, which results in less

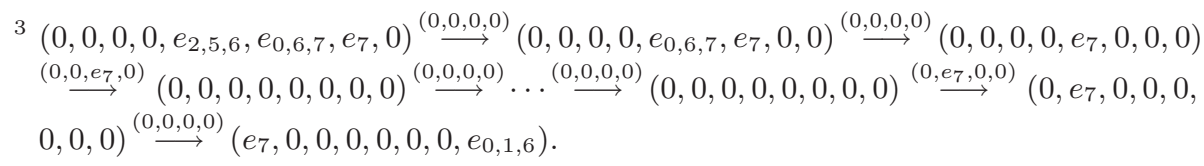


computations in the subsequent steps and may allow us to break more rounds by guessing the subkeys involved, depending on how many candidates are remaining.

The above analysis enables us to give a related-key rectangle attack on the first 26 rounds of HIGHT with the final transformation only, after noting that the same 64 user key bits are used in Rounds 1, 2, 25 and 26 as well as the final transformation. With a success probability of $80 \%$, the attack requires $2^{49.7}$ chosen plaintexts, and has a time complexity of $2^{121.37} 26$-round HIGHT computations. See the Appendix A for the detailed attack procedure.

\subsection{Related-Key Impossible Differential Attack on 28-Round HIGHT}

19-Round Related-Key Impossible Differentials. We exploit certain 19round related-key impossible differentials: $\left(e_{7}, 0,0,0,0,0,0,0\right) \nrightarrow(0,0,0,0,0,0,0$, $\left.e_{0, \sim}\right)$, where the user key difference $\left(\Delta \mathrm{MK}_{15}, \cdots, \Delta \mathrm{MK}_{1}, \Delta \mathrm{MK}_{0}\right)$ is $\left(0, e_{7}, 0, \cdots\right.$, 0 ), which start from Round 8 and end at Round 26. They are also built in a miss-in-the-middle manner: a 12-round related-key differential with probability 1 is concatenated with a 7 -round related-key differential with probability 1 , where the second right byte of the output difference of the 12-round related-key differential is $e_{\overline{0}}$, , and the second right byte of the difference of the 7 -round related-key differential is $e_{0, \sim}$, which contradict with each other.

Attacking Rounds 3-30. Similar to that given in Section 3.2, the 19-round related-key impossible differentials can be used to break the 28 rounds from Rounds 3 to 30 of HIGHT with only the final transformation; the main difference between them lies in that here we compute the related-key difference between a pair of data. The attack procedure is as follows.

1. Choose $2^{19}$ structures of $2^{40}$ plaintexts, where the two bytes $(0,1)$ and the least significant seven bits of the third bytes and the least significant bits of the fourth bytes of the $2^{40}$ plaintexts in a structure are fixed to certain values, and the other 40 bit positions take all the possible values. A structure proposes $2^{79}$ plaintext pairs $\left(P^{i}, \widetilde{P}^{j}\right)$ with difference $\left(?, ?, ?, ?, e_{\overline{0}, \sim}, e_{7}, 0,0\right)$, thus the $2^{19}$ structures propose a total of $2^{98}$ plaintext pairs with difference $\left(?, ?, ?, ?, e_{\overline{0}, \sim}, e_{7}, 0,0\right)$.

2. In a chosen-plaintext attack scenario, obtain all the ciphertexts of the plaintexts $P^{i}$ encrypted with $K_{A}$; we denote them by $C^{i}$, respectively; obtain all the ciphertexts of the plaintexts $\widetilde{P}^{j}$ encrypted with $K_{B}$; we denote them by $\widetilde{C}^{j}$, respectively, where $K_{A} \oplus K_{B}=\left(0, e_{7}, 0, \cdots, 0\right)$. Choose only the ciphertext pairs $\left(C^{i}, \widetilde{C}^{j}\right)$ with difference $\left(?, ?, ?, ?, e_{0, \sim}, 0,0,0\right)$.

3. Guess the two key bytes $\left(\mathrm{MK}_{0}, \mathrm{MK}_{3}\right)$, compute the subkeys $\left(\mathrm{WK}_{7}, \mathrm{SK}_{119}\right)$ in the final transformation and Round 30, and do the following.

(a) Partially decrypt every remaining ciphertext pair $\left(C^{i}, \widetilde{C}^{j}\right)$ with $\left(\mathrm{WK}_{7}\right.$, $\left.\mathrm{SK}_{119}\right)$ to get the two bytes $(7,6)$ of their intermediate values just before Round 30 . Check if they have a difference $(0, ?)$. Keep only the qualified pairs. 
(b) Guess the two key bytes $\left(\mathrm{MK}_{2}, \mathrm{MK}_{7}\right)$, compute the subkeys $\left(\mathrm{WK}_{6}, \mathrm{SK}_{118}\right)$ in the final transformation and Round 30, and compute the subkey $\mathrm{SK}_{114}$ in Round 29 with $\mathrm{MK}_{3}$ guessed above. Partially decrypt $\left(C^{i}, \widetilde{C}^{j}\right)$ with $\left(\mathrm{WK}_{4}, \mathrm{SK}_{114}, \mathrm{SK}_{118}\right)$ to get the two bytes $(5,4)$ of their intermediate values just before Round 29. Check if they have a difference $(0, ?)$. Keep only the qualified pairs.

(c) Guess the three key bytes $\left(\mathrm{MK}_{1}, \mathrm{MK}_{6}, \mathrm{MK}_{15}\right)$, compute the subkeys $\left(\mathrm{WK}_{5}, \mathrm{SK}_{95}, \mathrm{SK}_{117}\right)$ in the final transformation and Rounds 27 and 30, and compute the subkey $\mathrm{SK}_{113}$ in Round 29 with $\mathrm{MK}_{2}$ guessed above. Partially decrypt $\left(C^{i}, \widetilde{C}^{j}\right)$ with $\left(\mathrm{WK}_{5}, \mathrm{SK}_{95}, \mathrm{SK}_{113}, \mathrm{SK}_{117}\right)$ to get the two bytes $(3,2)$ of their intermediate values just before Round 28 . Check if they have a difference $(0, ?)$. Keep only the qualified pairs.

(d) Guess the two key bytes $\left(\mathrm{MK}_{5}, \mathrm{MK}_{14}\right)$, compute the subkeys $\left(\mathrm{SK}_{108}, \mathrm{SK}_{116}\right)$ in Rounds 28 and 30, and compute the subkeys $\left(\mathrm{WK}_{4}, \mathrm{SK}_{112}\right)$ in the final transformation and Round 29 with $\left(\mathrm{MK}_{0}, \mathrm{MK}_{1}\right)$ guessed above. For $l=0$ to 7 , do as follows.

- Guess the $l$-th bit $\mathrm{MK}_{10, l}$ of the key byte $\mathrm{MK}_{10}$, and compute the $(l+1)$ bits $\mathrm{SK}_{104,0-l}$ of the subkey $\mathrm{SK}_{104}$ in Round 27.

- For every remaining $\left(C^{i}, \widetilde{C}^{j}\right)$, Partially decrypt $C^{i}$ with $\left(\mathrm{WK}_{4}, \mathrm{SK}_{116}\right.$, $\left.\mathrm{SK}_{112}, \mathrm{SK}_{108}, \mathrm{SK}_{104,0-l}\right)$ to get its intermediate value $X_{26,1,0-l}^{i}$ just before Round 27 , and partially decrypt $\widetilde{C}^{j}$ with $\left(\mathrm{WK}_{4}, \mathrm{SK}_{116}, \mathrm{SK}_{112}\right.$, $\left.\mathrm{SK}_{108} \oplus e_{7}, \mathrm{SK}_{104,0-l}\right)$ to get its intermediate value $\widetilde{X}_{26,1,0-l}^{j}$ just before Round 27. Keep only the pairs such that $X_{26,1,0-l}^{i}=\widetilde{X}_{26,1,0-l}^{j}$.

4. For all the plaintext pairs $\left(P^{i}, \widetilde{P}^{j}\right)$ corresponding to remaining ciphertext pairs $\left(C^{i}, \widetilde{C}^{j}\right)$, do the following.

(a) For $l=0$ to 7 , do as follows.

- Guess the $l$-th bit $\mathrm{MK}_{11, l}$ of the key byte $\mathrm{MK}_{11}$, and compute the $(l+1)$ bits $\mathrm{SK}_{11,0-l}$ of the subkey $\mathrm{SK}_{11}$ in Round 3.

- Partially decrypt every remaining $\left(P^{i}, \widetilde{P}^{j}\right)$ with $\mathrm{SK}_{11,0-l}$ to get their intermediate values $\left(X_{3,7}^{i}, X_{3,0,0-l}^{i}\right)$ and $\left(\widetilde{X}_{3,7}^{j}, \widetilde{X}_{3,0,0-l}^{j}\right)$ just after Round 3. Keep the pairs such that $X_{3,0,0-l}^{i}=\widetilde{X}_{3,0,0-l}^{j}$.

(b) Compute the subkeys $\left(\mathrm{SK}_{10}, \mathrm{SK}_{15}\right)$ in Rounds 3 and 4 with $\left(\mathrm{MK}_{10}, \mathrm{MK}_{15}\right)$ guessed in Step 3. Partially decrypt $\left(P^{i}, \widetilde{P}^{j}\right)$ with $\left(\mathrm{SK}_{10}, \mathrm{SK}_{15}\right)$ to get the two bytes $(7,0)$ of their intermediate values just after Round 4 . Check if they have a difference $(?, 0)$. Keep only the qualified pairs.

(c) Guess the key byte $\mathrm{MK}_{9}$, compute the subkey $\mathrm{SK}_{9}$ in Round 3, and compute the subkeys $\left(\mathrm{SK}_{14}, \mathrm{SK}_{19}\right)$ in Rounds 4 and 5 with $\left(\mathrm{MK}_{2}, \mathrm{MK}_{14}\right)$ guessed in Step 3. Partially decrypt $P^{i}$ with $\left(\mathrm{SK}_{9}, \mathrm{SK}_{14}, \mathrm{SK}_{19}\right)$ to get the two bytes $(7,0)$ of its intermediate value just after Round 5 , and partially decrypt $\widetilde{P}^{j}$ with $\left(\mathrm{SK}_{9}, \mathrm{SK}_{14} \oplus e_{7}, \mathrm{SK}_{19}\right)$ to get the two bytes $(7,0)$ of its intermediate value just after Round 5 . Check if they have a difference $(?, 0)$. Keep only the qualified pairs.

(d) Guess the two key bytes $\left(\mathrm{MK}_{8}, \mathrm{MK}_{13}\right)$, compute the subkeys $\left(\mathrm{SK}_{8}, \mathrm{SK}_{13}\right)$ in Rounds 3 and 4, and compute the subkeys $\left(\mathrm{SK}_{18}, \mathrm{SK}_{29}\right)$ in Rounds 
5 and 6 with $\left(\mathrm{MK}_{1}, \mathrm{MK}_{6}\right)$ guessed in Step 3. Partially decrypt $\left(P^{i}, \widetilde{P}^{j}\right)$ with $\left(\mathrm{SK}_{8}, \mathrm{SK}_{13}, \mathrm{SK}_{18}, \mathrm{SK}_{29}\right)$ to get the two bytes $(7,0)$ of their intermediate values just after Round 6 . Check if they have a difference $\left(e_{0, \sim}, 0\right)$. Keep only the qualified pairs.

(e) Guess the key byte $\mathrm{MK}_{12}$, compute the subkey $\mathrm{SK}_{12}$ in Round 4, and compute the subkeys $\left(\mathrm{SK}_{17}, \mathrm{SK}_{22}, \mathrm{SK}_{27}\right)$ in Rounds 5, 6 and 7 with $\left(\mathrm{MK}_{0}, \mathrm{MK}_{5}, \mathrm{MK}_{10}\right)$ guessed in Step 3. For every remaining $\left(P^{i}, \widetilde{P}^{j}\right)$, partially decrypt the two bytes $(1,0)$ of their intermediate values just after Round 3 with $\left(\mathrm{SK}_{12}, \mathrm{SK}_{17}, \mathrm{SK}_{22}, \mathrm{SK}_{27}\right)$ to get the two bytes $(7,0)$ of their intermediate values just after Round 7 . Check if they have a difference $\left(e_{7}, 0\right)$. If none of the plaintext pairs satisfies this test, then record the guessed 120 key bits $\left(\mathrm{MK}_{0}, \cdots, \mathrm{MK}_{3}, \mathrm{MK}_{5}, \cdots, \mathrm{MK}_{15}\right)$, and execute Step 5; otherwise, discard this guess and try another.

5. For a recorded $\left(\mathrm{MK}_{0}, \cdots, \mathrm{MK}_{3}, \mathrm{MK}_{5}, \cdots, \mathrm{MK}_{15}\right)$, exhaustively search for the remaining 8 key bits with three known pairs of plaintexts and ciphertexts. If a 128-bit key is suggested, output it as the user key of the 28-round HIGHT; otherwise, go to Step 3.

There is a 25-bit filtering condition on the ciphertext pairs in Step 2, and a 8bit filtering condition in each of Steps 3-(a) (d) and Steps 4-(a) (e). Hence, for every key guess, it is expected about $2^{98} \cdot 2^{-25-8 \times 8}=2^{9}$ plaintext pairs remain after Step 4 - $(\mathrm{d})$, and about $2^{120} \cdot\left(1-2^{-8}\right)^{2^{9}} \approx 2^{120} \cdot e^{-2} \approx 2^{117.12}$ guesses of the 120 key bits are recorded in Step 4-(e). Thus, the expected number of suggested wrong keys in Step 5 is about $2^{-192} \cdot 2^{117.12+8}=2^{-66.88}$. Thus, the correct key can be determined.

Step 3 has about $2 \cdot 2^{73} \cdot 2^{16} \cdot \frac{1}{4} \cdot \frac{1}{28}+2 \cdot 2^{65} \cdot 2^{32} \cdot \frac{1}{4} \cdot \frac{2}{28}+2 \cdot 2^{57} \cdot 2^{56} \cdot \frac{1}{4} \cdot \frac{3}{28}+$ $\sum_{l=0}^{7}\left(2 \cdot 2^{49-l} \cdot 2^{72+l+1} \cdot \frac{1}{2} \cdot \frac{1}{4} \cdot \frac{4}{28}\right) \approx 2^{120.19}$ computations, where $\frac{1}{2}$ means the average fraction of the guessed keys that are tested in a step. Step 4 has about $\sum_{l=0}^{7}\left(2 \cdot 2^{41-l} \cdot 2^{80+l+1} \cdot \frac{1}{2} \cdot \frac{1}{4} \cdot \frac{1}{28}\right)+2 \cdot 2^{33} \cdot 2^{88} \cdot \frac{1}{2} \cdot \frac{1}{4} \cdot \frac{2}{28}+2 \cdot 2^{25} \cdot 2^{96} \cdot \frac{1}{2} \cdot \frac{1}{4} \cdot \frac{3}{28}+2$. $2^{17} \cdot 2^{112} \cdot \frac{1}{2} \cdot \frac{1}{4} \cdot \frac{4}{28}+2 \cdot 2^{120} \cdot\left[1+\left(1-2^{-8}\right)+\cdots+\left(1-2^{-8}\right)^{2^{9}}\right] \cdot \frac{1}{2} \cdot \frac{1}{4} \cdot \frac{4}{28} \approx 2^{124.79}$ computations. Step 5 has about $2^{125.12}$ computations. Therefore, the attack has a total time complexity of about $2^{125.99}$ 28-round HIGHT computations.

\section{Conclusions}

The HIGHT block cipher was proposed for low-resource devices at CHES '06. In this paper, we present an impossible differential attack on 25-round HIGHT, a related-key rectangle attack on 26-round HIGHT and a related-key impossible differential attack on 28-round HIGHT. Like most cryptanalytic attacks on block ciphers, the presented attacks are theoretical, but they suggest that the reduced versions of HIGHT are less secure than they should be. These are better than any previously known cryptanalytic results on HIGHT in terms of the numbers of attacked rounds. 


\section{Acknowledgments}

The author is very grateful to his supervisor Prof. Chris Mitchell for his editorial comments.

\section{References}

1. Biham, E.: New types of cryptanalytic attacks using related keys. In: Helleseth, T. (ed.) EUROCRYPT 1993. LNCS, vol. 765, pp. 398-409. Springer, Heidelberg (1994)

2. Biham, E., Biryukov, A., Shamir, A.: Cryptanalysis of Skipjack reduced to 31 rounds using impossible differentials. In: Stern, J. (ed.) EUROCRYPT 1999. LNCS, vol. 1592, pp. 12-23. Springer, Heidelberg (1999)

3. Biham, E., Biryukov, A., Shamir, A.: Miss in the middle attacks on IDEA and Khufu. In: Knudsen, L.R. (ed.) FSE 1999. LNCS, vol. 1636, pp. 124-138. Springer, Heidelberg (1999)

4. Biham, E., Dunkelman, O., Keller, N.: The rectangle attack - rectangling the Serpent. In: Pfitzmann, B. (ed.) EUROCRYPT 2001. LNCS, vol. 2045, pp. 340357. Springer, Heidelberg (2001)

5. Biham, E., Dunkelman, O., Keller, N.: Related-key boomerang and rectangle attacks. In: Cramer, R.J.F. (ed.) EUROCRYPT 2005. LNCS, vol. 3494, pp. 507-525. Springer, Heidelberg (2005)

6. Biham, E., Shamir, A.: Differential cryptanalysis of DES-like cryptosystems. In: Menezes, A.J., Vanstone, S.A. (eds.) CRYPTO 1990. LNCS, vol. 537, pp. 2-21. Springer, Heidelberg (1991)

7. Feldhofer, M., Dominikus, S., Wolkerstorfer, J.: Strong authentication for RFID systems using the AES algorithm. In: Joye, M., Quisquater, J.-J. (eds.) CHES 2004. LNCS, vol. 3156, pp. 357-370. Springer, Heidelberg (2004)

8. Feldhofer, M., Wolkerstorfer, J., Rijmen, V.: AES implementation on a grain of sand. IEE Proceedings on Information Security 152(1), 13-20 (2005)

9. Hong, D., Sung, J., Hong, S., Lim, J., Lee, S., Koo, B.-S., Lee, C., Chang, D., Lee, J., Jeong, K., Kim, H., Kim, J., Chee, S.: HIGHT: a new block cipher suitable for low-resource device. In: Goubin, L., Matsui, M. (eds.) CHES 2006. LNCS, vol. 4249, pp. 46-59. Springer, Heidelberg (2006)

10. Hong, S., Kim, J., Lee, S., Preneel, B.: Related-key rectangle attacks on reduced versions of SHACAL-1 and AES-192. In: Gilbert, H., Handschuh, H. (eds.) FSE 2005. LNCS, vol. 3557, pp. 368-383. Springer, Heidelberg (2005)

11. Kelsey, J., Kohno, T., Schneier, B.: Amplified boomerang attacks against reducedround MARS and Serpent. In: Schneier, B. (ed.) FSE 2000. LNCS, vol. 1978, pp. 75-93. Springer, Heidelberg (2001)

12. Kelsey, J., Schneier, B., Wagner, D.: Key-schedule cryptanalysis of IDEA, G-DES, GOST, SAFER, and Triple-DES. In: Koblitz, N. (ed.) CRYPTO 1996. LNCS, vol. 1109, pp. 237-251. Springer, Heidelberg (1996)

13. Kim, J., Kim, G., Hong, S., Lee, S., Hong, D.: The related-key rectangle attack - application to SHACAL-1. In: Wang, H., Pieprzyk, J., Varadharajan, V. (eds.) ACISP 2004. LNCS, vol. 3108, pp. 123-136. Springer, Heidelberg (2004)

14. Knudsen, L.R.: Trucated and higher order differentials. In: Preneel, B. (ed.) FSE 1994. LNCS, vol. 1008, pp. 196-211. Springer, Heidelberg (1995) 
15. Knudsen, L.R.: DEAL- a 128-bit block cipher, Technical report, Department of Informatics, University of Bergen, Norway (1998)

16. Lu, J., Kim, J., Keller, N., Dunkelman, O.: Related-key rectangle attack on 42round SHACAL-2. In: Katsikas, S.K., Lopez, J., Backes, M., Gritzalis, S., Preneel, B. (eds.) ISC 2006. LNCS, vol. 4176, pp. 85-100. Springer, Heidelberg (2006)

17. Lucks, S.: The saturation attack - a bait for Twofish. In: Matsui, M. (ed.) FSE 2001. LNCS, vol. 2355, pp. 1-15. Springer, Heidelberg (2002)

18. Matsui, M.: Linear cryptanalysis method for DES cipher. In: Helleseth, T. (ed.) EUROCRYPT 1993. LNCS, vol. 765, pp. 386-397. Springer, Heidelberg (1994)

19. National Institute of Standards and Technology (NIST). Advanced Encryption Standard (AES), FIPS-197 (2001)

20. Wagner, D.: The boomerang attack. In: Knudsen, L.R. (ed.) FSE 1999. LNCS, vol. 1636, pp. 156-170. Springer, Heidelberg (1999)

\section{A Procedure of the Related-Key Rectangle Attack on 26-Round HIGHT}

1. Choose $2^{14.7}$ structures $S_{i}$ of $2^{33}$ plaintexts $P_{i, l}$ each, $i=1,2, \cdots, 2^{14.7}$, $l=1,2, \cdots, 2^{33}$, where in each structure the 31 bit positions $(0-31)$ of $P_{i, l}$ are fixed, and the remaining 33 bit positions take all the possible values. In a chosen plaintext attack scenario, obtain the ciphertexts $C_{i, l}, C_{i, l}^{*}, C_{i, l}^{\prime}$ and $C_{i, l}^{\prime *}$ of $P_{i, l}$ encrypted with $K_{A}, K_{B}, K_{C}$ and $K_{D}$, respectively, where $K_{A} \oplus K_{B}=K_{C} \oplus K_{D}=\left(0, \cdots, 0, e_{7}, 0,0\right)$ and $K_{A} \oplus K_{C}=K_{B} \oplus K_{D}=$ $\left(0, e_{7}, 0, \cdots, 0\right)$.

2. Guess the 8 key bytes $\left(\mathrm{MK}_{0}, \cdots, \mathrm{MK}_{7}\right)$, compute the subkeys $\left(\mathrm{SK}_{0}, \cdots, \mathrm{SK}_{7}\right)$ in Rounds 1 and 2, and do as follows.

(a) Partially encrypt each plaintext $P_{i, l}$ through Rounds 1 and 2 with $\left(\mathrm{SK}_{0}\right.$, $\left.\cdots, \mathrm{SK}_{7}\right)$ to get its intermediate value $x_{i, l}$ just after Round 2 . Then, partially decrypt $x_{i, l} \oplus\left(e_{1,3,5}, e_{0,1,6}, e_{7}, 0,0,0,0,0\right)$ through Rounds 1 and 2 with $\left(\mathrm{SK}_{0}, \mathrm{SK}_{1}, \mathrm{SK}_{2} \oplus e_{7}, \mathrm{SK}_{3}, \cdots, \mathrm{SK}_{7}\right)$ to get its plaintext, denoted by $\widetilde{P}_{i, l}$. Find $\widetilde{P}_{i, l}$ in $S_{i}$. We denote by $\widetilde{C}_{i, l}, \widetilde{C}_{i, l}^{*}, \widetilde{C}_{i, l}^{\prime}$ and $\widetilde{C}_{i, l}^{* *}$ the corresponding ciphertexts for $\widetilde{P}_{i, l}$ encrypted under $K_{A}, K_{B}, K_{C}$ and $K_{D}$, respectively. This process generates $2^{14.7} \cdot 2^{33}=2^{47.7}$ plaintext pairs for every key guess, which can produce the difference $\left(e_{1,3,5}, e_{0,1,6}, e_{7}, 0,0,0\right.$, $0,0)$ just before Round 3 .

(b) Compute the subkeys $\left(\mathrm{SK}_{96}, \cdots, \mathrm{SK}_{99}\right),\left(\mathrm{SK}_{100}, \cdots, \mathrm{SK}_{103}\right)$ and $\left(\mathrm{WK}_{0}\right.$, $\left.\cdots, \mathrm{WK}_{3}\right)$ with $\left(\mathrm{MK}_{0}, \cdots, \mathrm{MK}_{7}\right)$. Then, partially decrypt all the $C_{i, l}$ and $C_{i, l}^{\prime}$ with these subkeys to get their respective intermediate values $T_{i, l}$ and $T_{i, l}^{\prime}$ just before Round 25 , and partially decrypt all the $\widetilde{C}_{i, l}^{*}$ and $\widetilde{C}_{i, l}^{*^{\prime}}$ with the related subkeys $\left(\mathrm{SK}_{96} \oplus e_{7}, \mathrm{SK}_{97}, \mathrm{SK}_{98}, \mathrm{SK}_{99}\right),\left(\mathrm{SK}_{100}, \cdots, \mathrm{SK}_{103}\right)$ and $\left(\mathrm{WK}_{0}, \mathrm{WK}_{1}, \mathrm{WK}_{2} \oplus e_{7}, \mathrm{WK}_{3}\right)$ to get their respective intermediate values $\widetilde{T}_{i, l}^{*}$ and $\widetilde{T}_{i, l}^{*^{\prime}}$ just before Round 25 . Store $\left(T_{i, l}, T_{i, l}^{\prime}, \widetilde{T}_{i, l}^{*}, \widetilde{T}_{i, l}^{*^{\prime}}\right)$ in a hash table. Finally, check if both $T_{i_{1}, l_{1}} \oplus T_{i_{2}, l_{2}}^{\prime}$ and $\widetilde{T}_{i_{1}, l_{1}}^{*} \oplus \widetilde{T}_{i_{2}, l_{2}}^{\prime *}$ have the form $\left(?, ?, ?, e_{\overline{0}, \sim}, e_{7}, e_{2, \sim}, e_{7}, ?\right)$, for $1 \leq i_{1} \leq i_{2} \leq 2^{14.7}$ and $1 \leq l_{1}, l_{2} \leq$ $2^{33}$. If 6 or more quartets $\left(T_{i_{1}, l_{1}}, \widetilde{T}_{i_{1}, l_{1}}^{*}, T_{i_{2}, l_{2}}^{\prime}, \widetilde{T}_{i_{2}, l_{2}}^{*^{\prime}}\right)$ pass this test, record them, and go to Step 3; otherwise, repeat Step 2 with another guess. 
3. For $l=0$ to 7 :

(a) Guess the $l$-th bit $\mathrm{MK}_{10, l}$ of the key byte $\mathrm{MK}_{10}$, and compute the $(l+1)$ bits $\mathrm{SK}_{95,0-l}$ of the subkey $\mathrm{SK}_{95}$ in Round 24.

(b) Partially decrypt the two bytes $(0,7)$ of every remaining $\left(T_{i_{1}, l_{1}}, \widetilde{T}_{i_{1}, l_{1}}^{*}, T_{i_{2}, l_{2}}^{\prime}\right.$, $\left.\widetilde{T}_{i_{2}, l_{2}}^{*^{\prime}}\right)$ with $\mathrm{SK}_{95,0-l}$ to get the least significant $(l+1)$ bits of the bytes (7) of their intermediate values just before Round 24, and check if the intermeidate $(l+1)$ bits of $T_{i_{1}, l_{1}}$ and $T_{i_{2}, l_{2}}^{\prime}$ have a zero difference, and the intermeidate $(l+1)$ bits of $\widetilde{T}_{i_{1}, l_{1}}^{*}$ and $\widetilde{T}_{i_{2}, l_{2}}^{*^{\prime}}$ also have a zero difference. If 6 or more quartets pass this test, record them; otherwise, repeat Step 3-(a) with another guess.

4. Guess the key byte $\mathrm{MK}_{9}$, and compute the subkey $\mathrm{SK}_{94}$ in Round 24; for $l=0$ to 7 , do as follows.

(a) Guess the $l$-th bit $\mathrm{MK}_{13, l}$ of $\mathrm{MK}_{13}$, and compute the $(l+1)$ bits $\mathrm{SK}_{90,0-l}$ of the subkey $\mathrm{SK}_{90}$ in Round 23.

(b) Partially decrypt the two bytes $(5,6)$ of every remaining $\left(T_{i_{1}, l_{1}}, \widetilde{T}_{i_{1}, l_{1}}^{*}, T_{i_{2}, l_{2}}^{\prime}\right.$, $\left.\widetilde{T}_{i_{2}, l_{2}}^{*^{\prime}}\right)$ with $\left(\mathrm{SK}_{94}, \mathrm{SK}_{90,0-l}\right)$ to get the least significant $(l+1)$ bits of the bytes (5) of their intermediate values just before Round 23, and check if the intermeidate $(l+1)$ bits of $T_{i_{1}, l_{1}}$ and $T_{i_{2}, l_{2}}^{\prime}$ have a zero difference, and the intermeidate $(l+1)$ bits of $\widetilde{T}_{i_{1}, l_{1}}^{*}$ and $\widetilde{T}_{i_{2}, l_{2}}^{*^{\prime}}$ have a zero difference as well. If 6 or more quartets pass this test, record them; otherwise, repeat Step 4-(a) with another guess (if all the guesses of $\mathrm{MK}_{13, l}$ are tested, repeat Step 4 with another guess of $\mathrm{MK}_{9}$ ).

5. Guess the least significant 3 bits $\mathrm{MK}_{15,0-2}$ of the key byte $\mathrm{MK}_{15}$; for $l=3$ to 7 , do as follows.

(a) Guess the $l$-th bit $\mathrm{MK}_{15, l}$ of $\mathrm{MK}_{15}$, and compute the $(l+1)$ bits $\mathrm{SK}_{92,0-l}$ of the subkey $\mathrm{SK}_{92}$ in Round 24.

(b) Partially decrypt the two bytes $(1,2)$ of every remaining $\left(T_{i_{1}, l_{1}}, \widetilde{T}_{i_{1}, l_{1}}^{*}, T_{i_{2}, l_{2}}^{\prime}\right.$, $\left.\widetilde{T}_{i_{2}, l_{2}}^{*^{\prime}}\right)$ with $\mathrm{SK}_{92,0-l}$ to get the least significant $(l+1)$ bits of the bytes (1) of their intermediate values just before Round 24, and check if the intermeidate $(l+1)$ bits of $T_{i_{1}, l_{1}}$ and $T_{i_{2}, l_{2}}^{\prime}$ have a zero difference, and the intermeidate $(l+1)$ bits of $\widetilde{T}_{i_{1}, l_{1}}^{*}$ and $\widetilde{T}_{i_{2}, l_{2}}^{*^{\prime}}$ have a zero difference as well. If 6 or more quartets pass this test, record them; otherwise, repeat Step 5-(a) with another guess.

6. Guess the key bytes $\left(\mathrm{MK}_{8}, \mathrm{MK}_{12}\right)$, compute the subkeys $\left(\mathrm{SK}_{93}, \mathrm{SK}_{89}\right)$, and compute the subkey $\mathrm{SK}_{85}$ with $\mathrm{MK}_{0}$ guessed in Step 2. Partially decrypt the two bytes $(3,4)$ of every remaining $\left(T_{i_{1}, l_{1}}, \widetilde{T}_{i_{1}, l_{1}}^{*}, T_{i_{2}, l_{2}}^{\prime}, \widetilde{T}_{i_{2}, l_{2}}^{*^{\prime}}\right)$ with $\left(\mathrm{SK}_{93}, \mathrm{SK}_{89}\right.$, $\left.\mathrm{SK}_{85}\right)$ to get the two bytes $(3,2)$ of their intermediate values just before Round 22 , and check if the intermeidate values of $T_{i_{1}, l_{1}}$ and $T_{i_{2}, l_{2}}^{\prime}$ have a difference $(0, ?)$, and the intermeidate values of $\widetilde{T}_{i_{1}, l_{1}}^{*}$ and $\widetilde{T}_{i_{2}, l_{2}}^{*^{\prime}}$ have a difference $(0, ?)$ as well. If 6 or more quartets pass this test, execute Step 7 with them; otherwise, repeat this step with another guess. Now, for every remaining $\left(T_{i_{1}, l_{1}}, \widetilde{T}_{i_{1}, l_{1}}^{*}\right.$, $\left.T_{i_{2}, l_{2}}^{\prime}, \widetilde{T}_{i_{2}, l_{2}}^{*^{\prime}}\right)$, we obtain their intermediate values just before Round 24 ; we denote them by $\left(Q_{i_{1}, l_{1}}, \widetilde{Q}_{i_{1}, l_{1}}^{*}, Q_{i_{2}, l_{2}}^{\prime}, \widetilde{Q}_{i_{2}, l_{2}}^{*^{\prime}}\right)$, respectively. 
7. Guess the key byte $\mathrm{MK}_{11}$, compute the subkey $\mathrm{SK}_{88}$ in Round 23, and compute the subkey $\mathrm{SK}_{84}$ with $\mathrm{MK}_{7}$ guessed in Step 2. Partially decrypt the two bytes $(1,2)$ of $\left(Q_{i_{1}, l_{1}}, \widetilde{Q}_{i_{1}, l_{1}}^{*}, Q_{i_{2}, l_{2}}^{\prime}, \widetilde{Q}_{i_{2}, l_{2}}^{*^{\prime}}\right)$ with $\left(\mathrm{SK}_{88}, \mathrm{SK}_{84}\right)$ to get the two bytes $(1,0)$ of their intermediate values just before Round 22 , and check if the intermediate values of $Q_{i_{1}, l_{1}}, Q_{i_{2}, l_{2}}^{\prime}$ have a difference belonging to the set $\left\{\left(x, e_{7}\right) \mid x \in \mathcal{S}\right\}$, and the intermediate values of $\widetilde{Q}_{i_{1}, l_{1}}^{*}$ and $\widetilde{Q}_{i_{2}, l_{2}}^{*^{\prime}}$ also have a difference belonging to the set $\left\{\left(x, e_{7}\right) \mid x \in \mathcal{S}\right\}$. If 6 or more quartets $\left(Q_{i_{1}, l_{1}}, \widetilde{Q}_{i_{1}, l_{1}}^{*}, Q_{i_{2}, l_{2}}^{\prime}, \widetilde{Q}_{i_{2}, l_{2}}^{*^{\prime}}\right)$ pass this test, execute Step 8 with them; otherwise, repeat this step with another guess of $\mathrm{MK}_{11}$.

8. Compute the subkey $\mathrm{SK}_{80}$ with $\mathrm{MK}_{3}$ guessed in Step 2. For every remaining $\left(Q_{i_{1}, l_{1}}, \widetilde{Q}_{i_{1}, l_{1}}^{*}, Q_{i_{2}, l_{2}}^{\prime}, \widetilde{Q}_{i_{2}, l_{2}}^{*^{\prime}}\right)$, since we already obtain the two bytes $(1,2)$ of their intermediate values just before Round 22, we can partially decrypt them with $\mathrm{SK}_{80}$ to check if the bytes (1) of the intermediate values just before Round 21 of $\left(Q_{i_{1}, l_{1}}, Q_{i_{2}, l_{2}}^{\prime}\right)$ have a zero difference, and the bytes (1) of the intermediate values just before Round 21 of $\left(\widetilde{Q}_{i_{1}, l_{1}}^{*}, \widetilde{Q}_{i_{2}, l_{2}}^{*^{\prime}}\right)$ have a zero difference as well. If 6 or more $\left(Q_{i_{1}, l_{1}}, \widetilde{Q}_{i_{1}, l_{1}}^{*}, Q_{i_{2}, l_{2}}^{\prime}, \widetilde{Q}_{i_{2}, l_{2}}^{*^{\prime}}\right)$ pass this test, record the guessed 120 key bits $\left(\mathrm{MK}_{0}, \cdots, \mathrm{MK}_{13}, \mathrm{MK}_{15}\right)$, and go to Step 9; otherwise, repeat Step 7 with another guess of $\mathrm{MK}_{11}$.

9. For a recorded $\left(\mathrm{MK}_{0}, \cdots, \mathrm{MK}_{13}, \mathrm{MK}_{15}\right)$, exhaustively search for the remaining 8 key bits with a known plaintext/ciphertext pair. If a 128-bit key is suggested, output it as the user key of the 26-round HIGHT; otherwise, go to Step 2 (If all the guesses are tested during any of Steps 3-8, repeat its previous steps with other guesses).

The related-key rectangle distinguisher involves four different keys, thus about $2^{47.7 \times 2}=2^{95.4}$ candidate quartets are constructed for every guess in Step 2. To produce the output difference $\delta^{\prime}$, the two pairs in a right quartet must have differences $\left(?, ?, ?, e_{\overline{0}, \sim}, e_{7}, e_{2, \sim}, e_{7}, ?\right)$ just before Round 25 , so a candidate quartet that does not meet this filtering condition is an incorrect quartet. Therefore, it is expected that almost all the $2^{64}$ guesses of $\left(\mathrm{MK}_{0}, \cdots, \mathrm{MK}_{7}\right)$ will pass Step 2 -(b), and for every guess about $2^{95.4} \cdot 2^{-20 \times 2}=2^{55.4}$ candidate quartets remain after 2-(b).

For every iteration in Step 3-(b), the probability that a quartet meets the filtering condition is $\left(2^{-1}\right)^{2}=2^{-2}$, so it follows that all the $2^{72}$ guesses of $\left(\mathrm{MK}_{0}, \cdots, \mathrm{MK}_{7}, \mathrm{MK}_{10}\right)$ will past Step 3, and for a wrong guess it is expected about $2^{55.4} \cdot 2^{-2 \times 8}=2^{39.4}$ quartets remain after Step 3. For every iteration in Step 4-(b), the probability that a quartet meets the filtering condition is also $2^{-2}$, so it is expected that all the $2^{88}$ guesses of $\left(\mathrm{MK}_{0}, \cdots, \mathrm{MK}_{7}, \mathrm{MK}_{9}, \mathrm{MK}_{10}, \mathrm{MK}_{13}\right)$ will past this step, and for a wrong guess about $2^{39.4} \cdot 2^{-2 \times 8}=2^{23.4}$ quartets remain after Step 4. For every iteration in Step 5-(b), the probability that a quartet meets the filtering condition is $2^{-2}$, so for a wrong guess about $2^{23.4} \cdot 2^{-2 \times 5}=2^{13.4}$ quartets remain after Step 5. In Step 6, the probability that a quartet meets the filtering condition is also $2^{-8 \times 2}=2^{-16}$, so for a wrong guess about $2^{13.4} \cdot 2^{-16}=2^{-2.6}$ quartets remain after Step 6, and the probability that 6 or more quartets pass the test for a wrong guess is approximately 
$\sum_{i=6}^{2^{13.4}}\left[\left(\begin{array}{c}2^{13.4} \\ i\end{array}\right) \cdot\left(2^{-16}\right)^{i} \cdot\left(1-2^{-16}\right)^{2^{13.4}-i}\right] \approx 2^{-25.09}$, thus it is expected that about $2^{112} \cdot 2^{-25.09}=2^{86.91}$ guesses of $\left(\mathrm{MK}_{0}, \cdots, \mathrm{MK}_{10}, \mathrm{MK}_{12}, \mathrm{MK}_{13}, \mathrm{MK}_{15}\right)$ pass Step 6. In Step 7, the probability that a quartet meets the filtering condition is $\left(\frac{24}{2^{7}}\right)^{2}=2^{-4.83}$, and the probability that 6 or more quartets pass the test for a wrong guess is approximately $\left(2^{-4.83}\right)^{6} \approx 2^{-28.98}$, so it is expected about $2^{86.91+8} \cdot 2^{-28.98}=2^{65.93}$ guesses of $\left(\mathrm{MK}_{0}, \cdots, \mathrm{MK}_{13}, \mathrm{MK}_{15}\right)$ pass Step 7. In Step 8 , the probability that 6 or more quartets pass the test for a wrong guess is approximately $\left(2^{-8 \times 2}\right)^{6}=2^{-96}$, thus it is expected about $2^{65.93} \cdot 2^{-96}=2^{-30.07}$ guesses of $\left(\mathrm{MK}_{0}, \cdots, \mathrm{MK}_{13}, \mathrm{MK}_{15}\right)$ pass Step 8. Therefore, it is expexcted that we can find the correct user key with $2^{8}$ trials in Step 9.

The attack requires $2^{49.7}$ related-key chosen plaintexts. Step 2-(a) has about $2 \cdot 2^{47.7} \cdot 2^{64} \cdot \frac{1}{2} \cdot \frac{2}{26} \approx 2^{108} 26$-round HIGHT computations, where $\frac{1}{2}$ means the average fraction of the guessed keys that are tested in the step. The time complexity of Step 2-(b) is dominated by the partial decryptions, which is about $4 \cdot 2^{47.7} \cdot 2^{64} \cdot \frac{1}{2} \cdot \frac{2}{26} \approx 2^{109}$ computations. Step 3 has about $\sum_{l=0}^{7}\left(4 \cdot 2^{55.4-2 \cdot l} \cdot 2^{65+l} \cdot \frac{1}{2}\right.$. $\left.\frac{1}{4} \cdot \frac{1}{26}\right) \approx 2^{115.69}$ computations. Step 4 has about $\sum_{l=0}^{7}\left(4 \cdot 2^{39.4-2 \cdot l} \cdot 2^{81+l} \cdot \frac{1}{2} \cdot \frac{1}{4} \cdot \frac{2}{26}\right) \approx$ $2^{116.69}$ computations. Step 5 has about $\sum_{l=0}^{4}\left(4 \cdot 2^{23.4-2 \cdot l} \cdot 2^{92+l} \cdot \frac{1}{2} \cdot \frac{1}{4} \cdot \frac{1}{26}\right) \approx 2^{110.65}$ computations. Step 6 has about $4 \cdot 2^{13.4} \cdot 2^{112} \cdot \frac{1}{2} \cdot \frac{1}{4} \cdot \frac{3}{26} \approx 2^{121.28}$ computations. Step 7 has about $4 \cdot 6 \cdot 2^{94.91} \cdot \frac{1}{2} \cdot \frac{1}{4} \cdot \frac{2}{26} \approx 2^{92.79}$ computations. Step 8 has about $4 \cdot 6 \cdot 2^{65.93} \cdot \frac{1}{2} \cdot \frac{1}{4} \cdot \frac{1}{26} \approx 2^{62.81^{2}}$ computations. Therefore, the attack has a total time complexity of about $2^{121.37} 26$-round HIGHT computations.

In Step 8, it is expected that about $2^{95.4} \cdot 2^{-92.4}=8$ quartets pass the filtering condition for the correct key, and the probability that 6 or more quartets pass the test for the correct key guess is approximately $\sum_{i=6}^{2^{95.4}}\left[\left(\begin{array}{c}2^{95.4} \\ i\end{array}\right) \cdot\left(2^{-92.4}\right)^{i} \cdot(1-\right.$ $\left.\left.2^{-92.4}\right)^{2^{95.4}-i}\right] \approx 0.8$, so this related-key rectangle attack can break the 26 -round HIGHT with a success probability of $80 \%$. 